\title{
Investigation of the Interface Between Biological Cell Tissue and Hard Substrate Materials using CrossBeam Technology
}

\author{
P. Gnauck*, C. Burkhardt**, H. Wolburg***, W. Nisch** \\ * Carl Zeiss SMT - Nano Technology Systems Division, 73447 Oberkochen, Germany \\ ** NMI, Markwiesenstr.55, 72760 Reutlingen, Germany \\ *** Institute of Pathology, University of Tuebingen, Germany
}

To investigate the embedding of implants into bone tissue and connective tissue, or the coupling of recording or stimulation electrodes to neural tissue, the precise knowledge of the interface (structure, chemical composition, internal layers, etc.) between cell tissue and implant or electrode material is essential. In electro-physiology the exact knowledge of the interface between cell tissue and stimulation or recording electrodes contributes to the interpretation of the recorded signals [1]. Furthermore the understanding of mechanisms influencing the behavior of cells on micro structured surfaces could help to optimize the surface of future implants.

The investigation of the interface between cell tissue and hard materials like silicon etc. is mostly limited to the investigation of epoxy replicas [3]. This is due to the limitations of the ultramicrotomy technique for cutting hard materials like silicon, glass, ceramics etc.

Other, previously used, techniques allowing to investigate the real interface in the TEM [2] are very time consuming and not very site specific.

To overcome the disadvantages of the microtome (only replicas), we have developed a fast preparation technique that allows a site specific investigation the real interface between silicon microstructures and cell tissue at high resolution in a CrossBeam (FIB / FESEM) instrument.

Fibroblasts were cultured on titanium coated Si microstructured Si wafers. The wafers were sterilized in $70 \%$ ethanol, exposed to UV light and incubated in tissue culture medium overnight before seeding the cells. The cells were then washed in buffer, fixed in $2.5 \%$ buffered glutaraldehyde ( $\mathrm{pH}$ 7.4) for $30 \mathrm{~min}$, postfixed in $1 \% \mathrm{OsO}_{4}$ and dehydrated in a series of ethanol. The $70 \%$ ethanol step was performed with ethanol saturated with uranyl acetate. After dehydration, the specimens were embedded in Araldite The prepared specimens were then cross sectioned by using a high resolution CrossBeam instrumentand imaged directly with this instrument at high resolution (figs. 1-4). Due to the combination of a high resolution field emission SEM for superb imaging capabilities and a high resolution FIB for cutting this technique allows site specific preparation and high resolution investigation of the internal interface at the $\mathrm{nm}$ level. The CrossBeam instrument has also been used to cut a thin lamella out of the substrate, which was subsequently used for STEM investigation inside the CrossBeam instrument. Fig. 5 shows an example of the STEM imaging capabilities of the CrossBeam tool. The image quality is comparable to a TEM image. Due to the relatively low accelerating voltage of only $30 \mathrm{kV}$ the images shows excellent contrast.

References

[1] A. Stett, S. Weiss, P. Gnauck, M. Stelzle, W. Nisch, H. Haemmerle and E. Zrenner, Investigative Ophthalmology \& Visual Science (Suppl.) 38:41, 1997. 
[2] P. Gnauck, W. Nisch, 18th Annual International Conference of the IEEE Engineering in Medicine and Biology Society, 31. October - 3. November 1996, Amsterdam, The Netherlands, Vol. 2, 686

[3] F. Pfeiffer, B. Herzog, D. Kern, L. Scheideler, J. Geis-Gerstorfer, H. Wolburg, Microelectronic Engineering 67-68 (2003) 913-922

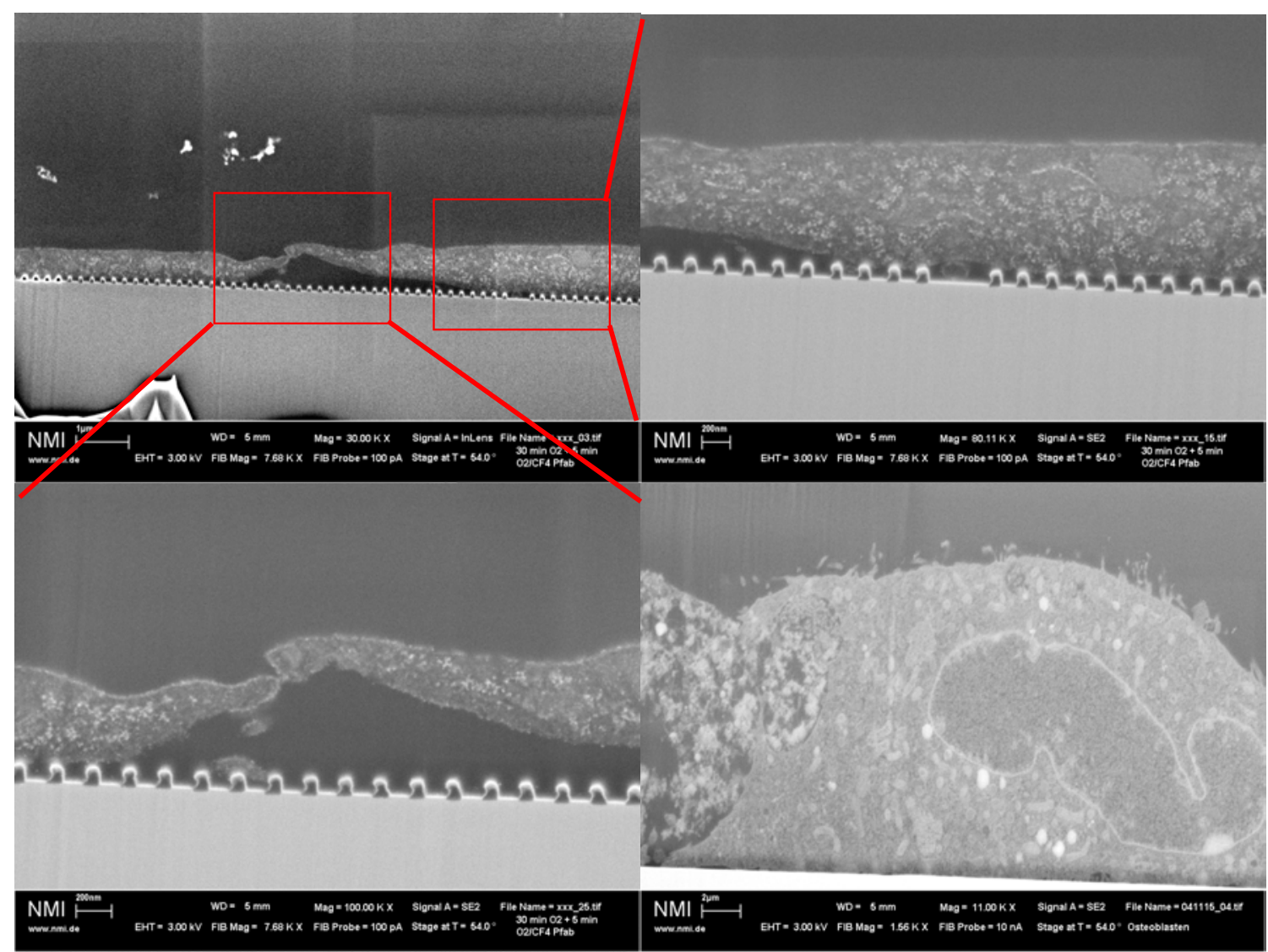

Fig. 1-4. Fibroblast cells grown on Si microstructures. The sample was cross-sectioned and imaged in SEM mode in a CrossBeam tool.

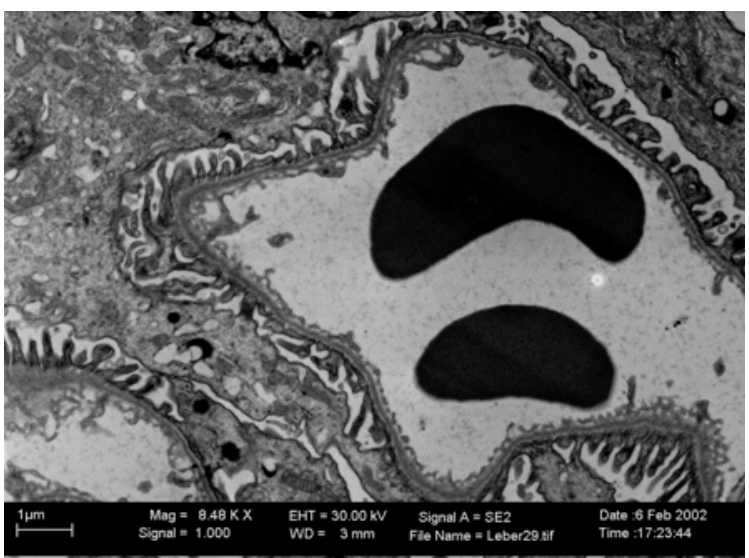

Fig. 5. 30kV STEM image of kidney cells 